\title{
Rate of Convergence of Discretization in Chebyshev Approximation
}

\author{
By C. B. Dunham and Jack Williams
}

\begin{abstract}
The paper treats, in a particularly simple fashion, the practical problem of the rate of convergence of discretization in real and complex Chebyshev approximation. Both linear and nonlinear approximations are discussed and, subject to certain conditions, quadratic convergence of the discretizations is obtained along with an explicit rate constant which can be estimated numerically.
\end{abstract}

1. Introduction. In their paper [4], Ellacott and Williams consider the problem of complex linear Chebyshev approximation involving discretization of a Jordan curve $C$, where $C$ consists of a finite number of smooth arcs. Specifically, let $f$, $\phi_{1}, \phi_{2}, \ldots, \phi_{n}$ be complex-valued continuous functions on $C$, where $\left\{\phi_{i}\right\}$ forms a basis for a subspace $P$. With $\|g\|=\max _{z \in C}|g(z)|$, we seek $p^{*} \in P$ for which

$$
\left\|f-p^{*}\right\| \leqslant\|f-p\| \text { for all } p \in P \text {. }
$$

It is known that a best approximation $p^{*}$ exists, and if $P$ is a Haar space, then $p^{*}$ is unique [5, Chapter 2]. As remarked in [4], approximation on the region enclosed by $C$ to those functions which are analytic there can be replaced by approximation on C.

In this note we are concerned with the case (of considerable practical interest) where the above problem is approached by replacing $C$ by a compact subset $Z_{k} \subset C$ and determining a best approximation $p_{k}$ to $f$ on $Z_{k}$. We then need to investigate to what extent the solution of this "descretized" problem approximates the solution of the original problem. When $Z_{k}$ is a finite point set, an algorithm for computing $p_{k}$ is described in [4].

2. Discretization Theory. In [4] the authors measure the quality of approximation in terms of $\mu\left(p_{k}\right)$, where

$$
\mu\left(p_{k}\right)=\left\|f-p^{*}\right\|-\max _{z \in Z_{k}}\left|f(z)-p_{k}(z)\right| .
$$

For practical purposes this does not provide complete information since the resulting approximation $p_{k}$ is used to approximate $f$ on $C$ where its error is given by $\left\|f-p_{k}\right\|$. Hence a more satisfactory measure of the accuracy of the discretization process is given by

$$
\rho\left(p_{k}\right)=\left\|f-p_{k}\right\|-\left\|f-p^{*}\right\| ;
$$

this quantity was introduced by Dunham [3e]. Suppose now that $p^{*}$ is a unique best approximation to $f$. Then, by a simple modification of the argument presented in 
Theorem 2.2 of [4], we obtain, subject to reasonable smoothness conditions, quadratic convergence to zero of $\rho\left(p_{k}\right)$.

ThEOREM. Let $C:=\{z: z=\gamma(t), t \in[0,1], \gamma(0)=\gamma(1)\}$ be a piecewise smooth Jordan curve consisting of the smooth arcs $C_{r}, r=1,2, \ldots, M$, and let $f$ and $\left\{\phi_{i}\right\}$ be twice continuously differentiable on each arc $C_{r}$. Let $Z_{k} \equiv Z_{k}(t) \subset C, k=$ $1,2, \ldots$, be a sequence of finite point subsets which each contain all the points of discontinuity of $d \gamma(t) / d t, t \in[0,1]$. If

$$
\left|Z_{k}\right|=\max _{\gamma\left(t_{2}\right) \in C} \min _{\gamma\left(t_{1}\right) \in Z_{k}} d\left(\gamma\left(t_{1}\right), \gamma\left(t_{2}\right)\right)
$$

satisfies $\left|Z_{k}\right| \rightarrow 0$ as $k \rightarrow \infty$, where

$$
d\left(\gamma\left(t_{1}\right), \gamma\left(t_{2}\right)\right)=\min \left\{\left|t_{1}-t_{2}\right|, 1-\left|t_{1}-t_{2}\right|\right\},
$$

then there exists a positive constant $K$ such that, for $k=1,2, \ldots$,

$$
\rho\left(p_{k}\right) \leqslant K\left|Z_{k}\right|^{2},
$$

where $p_{k}$ is a best approximation to $f$ on $Z_{k}$.

Proof. We consider $Z_{k} \subset C$ and let $h(z)=\left|f(z)-p_{k}(z)\right|^{2}$. Then, defining $R=$ $\operatorname{Re}\left(f-p_{k}\right), I=\operatorname{Im}\left(f-p_{k}\right)$, we have on $C$ the functions

$$
\begin{aligned}
h & =R^{2}+I^{2}, \quad h^{\prime}=2\left[R R^{\prime}+I I^{\prime}\right], \\
h^{\prime \prime} & =2\left[\left(R^{\prime}\right)^{2}+R R^{\prime \prime}+\left(I^{\prime}\right)^{2}+I I^{\prime \prime}\right],
\end{aligned}
$$

where differentiation is with respect to $t$. By examining the maximum of $h(\gamma(t))$ on $C$ and applying Taylor's theorem up to second derivatives, we obtain, as in [4], their inequality $(2.1)$

$$
\left\|f-p_{k}\right\|^{2} \leqslant \max _{z \in Z_{k}}\left|f(z)-p_{k}(z)\right|^{2}+\frac{1}{2} Q_{k}\left|Z_{k}\right|^{2},
$$

where $Q_{k}=\sup \left|d^{2} h(\gamma(t)) / d t^{2}\right|$, and the supremum is taken over $\gamma(t) \in C_{r}, r=$ $1,2, \ldots, M$. Hence,

$$
\begin{aligned}
\left\|f-p_{k}\right\|^{2}-\left\|f-p^{*}\right\|^{2} & <\max _{z \in Z_{k}}\left|f(z)-p_{k}(z)\right|^{2}-\left\|f-p^{*}\right\|^{2}+\frac{1}{2} Q_{k}\left|Z_{k}\right|^{2} \\
& <\frac{1}{2} Q_{k}\left|Z_{k}\right|^{2} .
\end{aligned}
$$

Therefore,

$$
\rho\left(p_{k}\right) \leqslant Q_{k}\left|Z_{k}\right|^{2} / 2\left(\left\|f-p_{k}\right\|+\left\|f-p^{*}\right\|\right)<Q_{k}\left|Z_{k}\right|^{2} / 4\left\|f-p^{*}\right\| .
$$

But, since $p_{k} \rightarrow p^{*}$ as $k \rightarrow \infty$ [2, Chapter 3], we have, for all $k, Q_{k}<K_{0}$ for some constant $K_{0}$, and the result follows.

Remark. The theorem also holds if $Z_{k}, k=1,2, \ldots$, are closed.

It is clear that the above theory can easily be adapted to treat real linear approximation on a real interval $C \equiv[a, b]$. In this case it is sufficient to have the points $a$ and $b$ contained in $Z_{k}$ for each $k=1,2, \ldots$; for practical approximation problems this is a natural requirement. Most important is the fact that, subject to certain conditions, it can also be applied to nonlinear, real or complex, Chebyshev approximations. For suppose $V$ is a class of approximating functions of the form $F(a, z)$, where $a=\left(a_{1}, a_{2}, \ldots, a_{n}\right)$, and is restricted such that $V$ consists of continuous functions on $C$. As is clear from the above proof, the critical requirement, apart from smoothness, is that the quantities $\left\{Q_{k}\right\}$ are uniformly bounded; 
in the linear case this follows from the uniform boundedness of the coefficients of $\left\{p_{k}\right\}$. Starting with the hypothesis that the best approximations $F\left(a_{k}, z\right) \in V$ on $Z_{k}$ exist and converge uniformly to $F\left(a^{*}, z\right)$, the best approximation on $C$, it is sufficient to assume that the first and second derivatives (with respect to $t$ ) of the real and imaginary parts of $F\left(a_{k}, z\right), z=\gamma(t) \in C_{r}, r=1,2, \ldots, M$, are uniformly bounded. Uniform boundedness of $Q_{k}$ then follows directly. As an example, consider real approximation by generalized rational functions

$$
p / q=\sum_{i=1}^{n} a_{i} \phi_{i}(x) / \sum_{i=1}^{m} a_{n+i} \psi_{i}(x)
$$

on $C$ a real interval $[a, b]$; we require

Assumption. $f$ has a unique best approximation $p^{*} / q^{*}$ on $C ; p^{*} / q^{*}$ has a unique representation under the normalization $\sum_{i=1}^{m}\left|a_{n+i}\right|=1$ and $q^{*}>0$ on $C$. Also, for $k=1,2, \ldots, a, b \in Z_{k}$. From [3f] a best approximation $p_{k} / q_{k}$ exists on $Z_{k}$ for $k$ sufficiently large and $p_{k} \rightarrow p^{*}, q_{k} \rightarrow q^{*}$ as $k \rightarrow \infty$. Hence (subject to appropriate differentiability of the $\left\{\phi_{i}(x)\right\}$ and $\left.\left\{\psi_{i}(x)\right\}\right)$, examining the expressions for $\left(p_{k} / q_{k}\right)^{\prime}$ and $\left(p_{k} / q_{k}\right)^{\prime \prime}$, it easily follows that the quantity $Q_{k}$ (contained in the proof of the above theorem) tends to a limit, and the result (2.1) holds.

In general, however, we note that uniform convergence of $F\left(a_{k}, z\right) \rightarrow F\left(a^{*}, z\right)$ cannot be guaranteed even if a best approximation $F\left(a^{*}, z\right)$ is unique; see for example [3a], [3b], [3d] for real nonlinear approximation and apply the results of Saff and Varga [6] for when best real rational approximations are best complex rational approximations. In practice, the corresponding functions $f$, for which this uniform convergence does not occur, may be unusual. In particular, for alternating approximation, the best approximation is degenerate [3c, Theorem 6] and the set of such $f$ may be nowhere dense [3c, Theorem 9].

Returning to the linear case, for many practical approximation problems, the measure of approximation given by $\rho\left(p_{k}\right)$ is usually sufficient, yet, even if the result (2.1) holds, it is possible that, with respect to the density $\left|Z_{k}\right|$, we have the equivalent phenomenon:

(i) the coefficients of $p_{k}$ do not converge quadratically to the coefficients of $p^{*}$ as $k \rightarrow \infty$,

(ii) $\left\|p_{k}-p^{*}\right\|$ does not converge quadratically to zero as $k \rightarrow \infty$.

Example. Consider the example of real approximation given in Cheney's text [2, p. 82] where $f(x)=x^{2}-1, C=[-1,1]$, and the approximating functions are of the form $p=a x, a \in \mathbf{R}$. Cheney shows that $p^{*} \equiv 0$ is the unique best approximation to $f$ on $C$; in fact, if $|a|<1$, then the maximum of $|f(x)-a x|$ occurs at $a / 2$ and has the value $1+a^{2} / 4$. Let $Z_{k}:=[-1,-2 / k] \cup[1 / k, 1], k=2,3, \ldots$ Then, since the function $x$ satisfies the Haar condition on each $Z_{k}$, best approximations on $Z_{k}$ are unique. Applying Komogorov's characterization theorem [5, Chapter 2] yields that $p_{k}$ is best on $Z_{k}$ if and only if $\operatorname{sign}(x)[f(x)-a x]$ alternates at least once on $Z_{k}$, from which it can easily be calculated (with the aid of a sketch) that $p_{k}=-x / k$; the extremal set consists of the two inner "end" points $-2 / k$ and $1 / k$. Hence, $\left\|f-p^{*}\right\|=1,\left\|f-p_{k}\right\|=1+1 / 4 k^{2}$, and $\left\|p_{k}-p^{*}\right\|=1 / k$; since $\left|Z_{k}\right|=$ $3 / 2 k$, the result (2.1) is satisfied subject to the above behavior (i) and (ii). Making $Z_{k}$ a finite subset with the same endpoints does not change the results. As a best 
real linear approximation is also a best complex linear approximation [6], the above example applies to complex approximation as well.

If $p^{*}$ is a strongly unique best approximation to $f$, that is, there exists a constant $\gamma>0$ such that

$$
\|f-p\|-\left\|f-p^{*}\right\|>\gamma\left\|p-p^{*}\right\| \quad \forall p \in P,
$$

then, clearly, $\left\|p_{k}-p^{*}\right\|$ tends to zero at least as fast as $\rho\left(p_{k}\right)$ since

$$
\left\|p_{k}-p^{*}\right\|<\rho\left(p_{k}\right) / \gamma \text {. }
$$

The above example shows that for $p^{*}$ merely unique a slower rate of convergence is possible. Also, the example of Dunham [3e] shows that the order of convergence in (2.1) cannot be improved by further smoothness hypotheses. For a treatment of the rate of convergence in Chebyshev approximation which employs strong uniqueness (real and complex case) see Chalmers [1] and the associated references for work on strong uniqueness.

Finally, it is important to note (as mentioned in [4]) that, because of its explicit nature, the rate constant $K$ occurring in (2.1) can in practice be estimated numerically. To see this we first observe from the proof that

$$
\rho\left(p_{k}\right)<\frac{Q_{k}}{4\left\|f-p^{*}\right\|}\left|Z_{k}\right|^{2} .
$$

In a practical situation it is, of course, the relative size of $\rho\left(p_{k}\right)$ which is of importance; agreement of $\left\|f-p_{k}\right\|$ with $\left\|f-p^{*}\right\|$ to two or three figures being good enough for many problems. Now

$$
\begin{aligned}
\frac{\rho\left(p_{k}\right)}{\left\|f-p^{*}\right\|} & \leqslant \frac{Q_{k}}{4 \max _{z \in Z_{k}}\left|f(z)-p^{*}(z)\right|^{2}}\left|Z_{k}\right|^{2} \\
& \leqslant \frac{Q_{k}}{4 \max _{z \in Z_{k}}\left|f(z)-p_{k}(z)\right|^{2}}\left|Z_{k}\right|^{2} .
\end{aligned}
$$

Now $Q_{k}$ can be estimated with the aid of simple difference approximations to $d^{2} h / d t^{2}$ on each $C_{r}, r=1,2, \ldots, M$, and, since $p_{k}(z)$ has been computed, its maximum error on $Z_{k}$ is immediately available. Hence, on the basis of an estimate for the bound (2.2), it would be possible to decide if $p_{k}$ is an acceptable replacement of $p^{*}$ and if not a new point set $Z_{k}$ should be selected for which $\left|Z_{k}\right|$ is correspondingly reduced. Many discretization results do not give any explicit rate constants; see, for example, [1]. We observe, however, that in general the rate constant occurring in (2.2) is not asymptotically correct (due to the simple inequalities used in its construction). Applying (2.2) to the given example of real approximation, we obtain

$$
\begin{gathered}
\rho\left(p_{k}\right)=1 / 4 k^{2}, \quad\left\|f-p^{*}\right\|=1 \\
\max _{z \in Z_{k}}\left|f(z)-p_{k}(z)\right|^{2}=\left(1-2 / k^{2}\right)^{2} \\
Q_{k}=8+\frac{12}{k}+\frac{2}{k^{2}} \text { (computed exactly), } \quad\left|Z_{k}\right|^{2}=9 / 4 k^{2}
\end{gathered}
$$


Hence (2.2) yields the upper bound

$$
\frac{9}{2 k^{2}}\left(1+\frac{3}{2 k}+\frac{1}{4 k^{2}}\right)\left(1-\frac{2}{k^{2}}\right)^{-2} \text {. }
$$

The value of $\rho\left(p_{k}\right) /\left\|f-p^{*}\right\|$ is $1 / 4 k^{2}$.

Department of Computer Science

University of Western Ontario

London, Ontario, Canada N6A 5B9

Department of Mathematics

University of Manchester

Manchester M13 9PL, England

1. B. A. Chalmers, "On the rate of convergence of discretization in Chebyshev approximation," SIAM J. Numer. Anal., v. 15, 1978, pp. 612-617.

2. E. W. CHENEY, Introduction to Approximation Theory, McGraw-Hill, New York, 1966.

3a. C. B. Dunham, "Rational approximation on subsets," J. Approx. Theory, v. 1, 1968, pp. 484-487.

3b. C. B. DunhaM, "Approximation by alternating families on subsets," Computing, v. 9, 1972, pp. 261-265.

3c. C. B. Dunham, "Alternating Chebyshev approximation," Trans. Amer. Math. Soc., v. 178, 1973, pp. 95-109.

3d. C. B. DUNham, "Varisolvent Chebyshev approximation on subsets," in Approximation Theory (G. G. Lorentz, Ed.), Academic Press, New York, 1973, pp. 337-340.

3e. C. B. Dunham, "Efficiency of Chebyshev approximation on finite subsets," J. Assoc. Comput. Mach., v. 21, 1974, pp. 311-313.

3f. C. B DunhaM, "Dependence of best rational Chebyshev approximations on the domain," Canad. Math. Bull., v. 20, 1977, pp. 451-454.

4. S. Ellacott \& JACK Williams, "Linear Chebyshev approximation in the complex plane using Lawson's algorithm," Math. Comp., v. 30, 1976, pp. 35-44.

5. G. G. LoReNTZ, Approximation of Functions, Holt, Rinehart and Winston, New York, 1966.

6. E. B. SAFF \& R. S. VARGA, "Nonuniqueness of best approximating complex rational functions," Bull. Amer. Math. Soc., v. 83, 1977, pp. 375-377. 